\title{
A VARIATION OF THE INTRINSIC MULTIPLE-SCALE HARMONIC BALANCE METHOD
}

BY

\author{
A. S. ATADAN
}

Dept. of Electrical and Electronics Engineering, P.O. Box 33, Muscat 123, Oman

\begin{abstract}
A variation of the intrinsic multiple-scale harmonic balance method is introduced by combining the intrinsic multiple-scale harmonic balance method with the ideas introduced to modify the method of multiple-scales. The combined method has the advantage of having the desirable characteristics of both techniques. This is demonstrated by solving the Duffing equation.
\end{abstract}

Introduction. The classical harmonic balance technique is a relatively simple method for investigating nonlinear oscillations. However, it has serious shortcomings and therefore is justifiably discarded by some authors [1]. The shortcoming of the classical harmonic balance method was later eliminated by Atadan and Huseyin to give consistent results $[2,3]$. More recently, a combination of the intrinsic harmonic balancing technique and the multiple-time-scales (derivative expansion) method was introduced by Huseyin and $\operatorname{Lin}[4]$.

In a separate development, a modification of the derivative expansion method was presented by Veronis [5]. This was done by replacing the natural frequency of the given equation by a series representation of frequency.

In this paper, a combination of the intrinsic multiple-scale harmonic balance method introduced by Huseyin and Lin [4], and the modification of the derivative expansion method given by Veronis [5] are presented. The two methods [4,5] are combined in such a way that the proposed technique has the desirable characteristics of both methods.

Firstly, it is conceptually simple and does not involve secular terms as in the method presented by Veronis [5] (see, e.g., Eq. (14) in [5]). The absence of secular terms follows from the intrinsic method of harmonic balancing $[2,3]$ which is the foundation of the intrinsic multiple-scale harmonic balancing technique [4] used here. Secondly, the frequency-parameter relationship obtained is in the form introduced by Veronis [5]. However, this is achieved by introducing a time-warping transformation rather than expanding the natural frequency as in Veronis [5]. For comparison reasons the method is introduced by solving the Duffing equation as in Veronis [5].

Received March 29, 1993.

1991 Mathematics Subject Classification. Primary 34C15; Secondary 34E15.

This work was partially supported by the NSERC of Canada under Grant OGPIN013.

(C)1996 Brown University 
Derivative expansion method and its modification. First, consider the following linear harmonic oscillator with a small damping:

$$
\frac{d^{2}}{d t^{2}} u+2 \varepsilon \frac{d}{d t} u+\omega_{0}^{2} u=0
$$

The (closed-form) solution of the linear problem (1) can be expressed as

$$
u=\frac{1}{2} a e^{-\varepsilon t} e^{i \sqrt{\omega_{0}^{2}-\varepsilon^{2} t}+\beta}+\text { c.c. }
$$

where c.c. indicates complex conjugate. Since the developments in this paper involve the derivative expansion method, we obtain the solution of the same problem via the derivative expansion method:

$$
u=\frac{1}{2} a e^{-\varepsilon t} e^{i \omega_{0}\left(1-\varepsilon^{2} / 2 \omega_{0}^{2}\right) t}+\text { c.c. }
$$

where the number of scales is limited to $T_{0}, T_{1}$, and $T_{2}$ as in references $[5,6]$.

In the case of the modified derivative expansion method introduced by Veronis [5], however, the solution given by (2) is obtained when the time-scales are limited to $T_{0}, T_{1}$, and $T_{2}$ as was done to derive (3). Veronis achieves this by replacing $\omega_{0}$ in Eq. (1) with a power series of the form

$$
\omega_{0}^{2}=\omega^{2}+\varepsilon \Omega_{1}+\varepsilon^{2} \Omega_{2}+\cdots
$$

Consider now the Duffing equation

$$
\frac{d^{2}}{d t^{2}} u+\omega_{0}^{2} u=\varepsilon \alpha u^{3}=0
$$

where the odd nonlinearities represent a soft or hard spring depending on the sign of the coefficient of $u^{3}$. Solution of the Duffing equation using the derivative expansion method can be obtained as [6]:

$$
\begin{aligned}
u= & \frac{1}{2} a e^{i(\omega t+\chi)}+\varepsilon \alpha a^{3} \frac{1}{64 \omega_{0}^{2}}\left[1-\varepsilon \alpha a^{2} \frac{21}{32 \omega_{0}^{2}}+\cdots\right] e^{i 3(\omega t+\chi)} \\
& +\varepsilon^{2} \alpha^{2} a^{5} \frac{1}{2048 \omega_{0}^{4}} e^{i 5(\omega t+\chi)}+\text { c.c. }+\cdots
\end{aligned}
$$

where the frequency is

$$
\omega=\omega_{0}+\varepsilon \alpha a^{2} \frac{3}{8 \omega_{0}}-\varepsilon^{2} \alpha^{2} a^{4} \frac{15}{256 \omega_{0}^{3}}+\cdots .
$$

Following Veronis [5], however, the frequency is obtained as

$$
\omega^{2}=\omega_{0}^{2}+\varepsilon \alpha a^{2} \frac{3}{4}\left(1+\varepsilon \alpha a^{2} \frac{1}{32 \omega_{0}^{2}}\right)+\cdots
$$

where the expression (4) has been substituted into the Duffing equation given by (5). 
A variation of the intrinsic multiple-scale harmonic balance method. In this section we will introduce a variation of the intrinsic multiple-scale harmonic balance method presented by Huseyin and Lin [4]. To start the development we seek an approximate solution to the Duffing equation given by (5) as

$$
u=u(t ; \varepsilon)
$$

which, warping the time via

$$
\tau=\omega(\varepsilon) t
$$

becomes

$$
u=u(\tau ; \varepsilon)
$$

Following (10) and (11), the Duffing equation can be rewritten as

$$
\omega^{2}(\varepsilon) \Delta^{2} u(\tau ; \varepsilon)+\omega_{0}^{2} u(\tau ; \varepsilon)+\varepsilon \alpha u^{3}=0
$$

where $\Delta$ is the derivative operator indicating differentiation with respect to $\tau$. Note that in $(12) \omega_{0}$ is not replaced by $\omega(\varepsilon)$ as was done by Veronis [5], but the frequency of solution is brought into the equation explicitly via the transformation (10).

Following the derivative expansion method, the scaled times are written as

$$
\tau_{n}=\frac{1}{n !} \varepsilon^{n} t, \quad n=0,1,2, \ldots
$$

where the coefficient of $\varepsilon^{n}$ is chosen as $(1 / n$ !) for reasons that will become clear shortly.

Introducing the time scaling into Eq. (12) and letting $\Omega(\varepsilon)=\omega^{2}(\varepsilon)$ results in

$$
\Omega(\varepsilon) \Delta^{2}(\varepsilon) u\left(\tau_{0}, \tau_{1}, \tau_{2}, \ldots ; \varepsilon\right)+\omega_{0}^{2} u\left(\tau_{0}, \tau_{1}, \tau_{2}, \ldots ; \varepsilon\right)+\varepsilon \alpha u^{3}\left(\tau_{0}, \tau_{1}, \tau_{2}, \ldots ; \varepsilon\right)=0
$$

The Taylor expansions of the frequency $\Omega(\varepsilon)$ and the derivative operator $\Delta(\varepsilon)$ are expressed as

$$
\Omega(\varepsilon)=\omega_{0}^{2}+\sum_{n=1}^{N} \varepsilon^{n} \frac{1}{n !} \Omega_{n}
$$

and

$$
\Delta(\varepsilon)=\sum_{n=0}^{N} \varepsilon^{n} \frac{1}{n !} \Delta_{n}
$$

where

$$
\Delta_{n} \equiv \frac{\partial}{\partial \tau_{n}}
$$

Following (16) one has

$$
\Delta^{2}(\varepsilon)=\Delta_{0}^{2}+\varepsilon 2 \Delta_{0} \Delta_{1}+\varepsilon^{2}\left[\Delta_{0} \Delta_{2}+\Delta_{1}^{2}\right]+\cdots
$$

It should be pointed out here that, rather than substituting the Taylor series expansion of the derivative operator into (14) as was done in [4], the perturbation equations are 
generated by differentiating (14) with respect to $\varepsilon$. Note that differentiation is the reason why $(1 / n !)$ is introduced in the time scales given by (13).

Following Huseyin [7-9], here the perturbation equations are generated by differentiation. The significance of generating perturbation equations by differentiation is that the number of perturbation equations need not be decided in advance. The standard perturbation techniques, however, do not have this advantage because they involve the substitution of a Taylor series expansion into the given differential equation.

Following this, the solution of Eq. (14) is assumed as

$$
\begin{aligned}
u & =u\left(\tau_{0}, \tau_{1}, \tau_{2} ; \varepsilon\right) \\
& =\sum_{m=-M}^{+M} a_{(m)}\left(\tau_{1}, \tau_{2}, ; \varepsilon\right) e^{i m \tau_{0}}
\end{aligned}
$$

where, in order to compare the results obtained in this paper to those of Veronis [5] and Nayfeh [6], the number of time scales is limited to $\tau_{0}, \tau_{1}$, and $\tau_{2}$ (i.e., $n=0,1,2$ ). The coefficient $a_{(m)}$ in series (19) is complex and given in the exponential form

$$
a_{(m)}\left(\tau_{1}, \tau_{2} ; \varepsilon\right)=d_{(m)}\left(\tau_{1}, \tau_{2} ; \varepsilon\right) e^{i m \phi\left(\tau_{1}, \tau_{2} ; \varepsilon\right)}
$$

with $d_{(m)} \in \Re$ and $\phi_{(m)} \in \mathfrak{R}$.

Evaluating (14) at $\varepsilon=0$ results in the zero-order perturbation equation

$$
\left(\Delta_{0}^{2}+1\right) u_{0}=0
$$

Differentiating (14) with respect to $\varepsilon$ as many times as required and evaluating at $\varepsilon=0$ yields the hierarchy of remaining perturbation equations, namely,

$$
\begin{aligned}
\left(\Delta_{0}^{2}+1\right) u_{1}= & -\left[\omega_{0}^{-2} \Omega_{1} \Delta_{0}+2 \Delta_{1}\right] \Delta_{0} u_{0}-\omega_{0}^{-2} \alpha u_{0}^{3} \\
\left(\Delta_{0}^{2}+1\right) u_{2}= & -\left[\omega_{0}^{-2} \Omega_{2} \Delta_{0}+2 \Delta_{2}\right] \Delta_{0} u_{0}-2\left[2 \omega_{0}^{-2} \Omega_{1} \Delta_{0}+\Delta_{1}\right] \Delta_{1} u_{0} \\
& -2\left[\omega_{0}^{-2} \Omega_{1} \Delta_{0}+2 \Delta_{1}\right] \Delta_{0} u_{1}-3 \omega_{0}^{-2} \alpha u_{0}^{2} u_{1}
\end{aligned}
$$

From the perturbation Eq. (21) one obtains

$$
\left(1-m^{2}\right) a_{0 .(m)}\left(\tau_{1}, \tau_{2}\right)=0,
$$

which yields

$$
a_{0,(m)}\left(\tau_{1}, \tau_{2}\right)=0 \text { for } m \neq \pm 1 .
$$

Following (25) and $a_{0,(-1)}\left(\tau_{1}, \tau_{2}\right)=a_{0,(+1)}^{*}\left(\tau_{1}, \tau_{2}\right)$, the solution of $(21)$ can be written as

$$
u_{0}\left(\tau_{0}, \tau_{1}, \tau_{2}\right)=a_{0,(+1)}\left(\tau_{1}, \tau_{2}\right) e^{i \tau_{0}}+\text { c.c. }
$$

where the superscript “*” indicates the complex conjugate. 
Substituting (26) into the r.h.s. of the first-order perturbation equation given by (22) results in

$$
\sum_{m=-\Lambda}^{+M}\left(1-m^{2}\right) a_{1,(m)}\left(\tau_{1}, \tau_{2}\right) e^{i m \tau_{0}}=M e^{i \tau_{0}}+N e^{i 3 \tau_{0}}+\text { c.c. }
$$

where

$$
\begin{aligned}
M & =\omega_{0}^{-2} \Omega_{1} a_{0,(+1)}-i 2 a_{0,(+1) \tau_{1}}-3 \alpha \omega_{0}^{-2} a_{0,(+1)}^{*} a_{0,(+1)}^{2}, \\
N & =-\alpha \omega_{0}^{-2} a_{0,(+1)}^{3}
\end{aligned}
$$

and

$$
a_{0,(+1)}=a_{0,(+1)}\left(\tau_{1}, \tau_{2}\right)
$$

Coefficients of $e^{i m \tau}(m \neq \pm 1, \pm 3)$ and $e^{i \tau}, e^{i 3 \tau}$ can be obtained from (27) as

$$
\begin{gathered}
a_{1,(m)}=0 \text { for } m \neq \pm 1, \pm 3 \\
\omega_{0}^{-2} \Omega_{1} a_{0,(+1)}\left(\tau_{1}, \tau_{2}\right)-i 2 a_{0,(+1) \tau_{1}}\left(\tau_{1}, \tau_{2}\right)-3 \alpha \omega_{0}^{-2} a_{0,(+1)}^{*}\left(\tau_{1}, \tau_{2}\right) a_{0,(+1)}^{2}\left(\tau_{1}, \tau_{2}\right)=0
\end{gathered}
$$

and

$$
8 a_{1,(3)}\left(\tau_{1}, \tau_{2}\right)=\alpha \omega_{0}^{-2} a_{0,(+1)}^{3}\left(\tau_{1}, \tau_{2}\right)
$$

respectively.

Substituting

$$
a_{0,(+1)}=a_{0,(+1)}\left(\tau_{1}, \tau_{2}\right)=\frac{1}{2} a_{0}\left(\tau_{1}, \tau_{2}\right) e^{i \phi_{0}\left(\tau_{1}, \tau_{2}\right)}
$$

into (32), and separating real and imaginary parts leads to

$$
\frac{\partial}{\partial \tau_{1}} a_{0}\left(\tau_{1}, \tau_{2}\right)=0
$$

Assuming that $a_{0}\left(\tau_{1}, \tau_{2}\right) \neq 0$,

$$
\frac{\partial}{\partial \tau_{1}} \phi_{0}\left(\tau_{1}, \tau_{2}\right)=-\frac{1}{2} \omega_{0}^{-2} \Omega_{1}+\frac{3}{8} \alpha \omega_{0}^{-2} a_{0}^{2}\left(\tau_{1}, \tau_{2}\right) .
$$

The steady-state solutions of (35) and (36) result in

$$
\begin{aligned}
a_{0} & =a_{0}\left(\tau_{2}\right), \\
\phi_{0} & =\phi_{0}\left(\tau_{2}\right), \\
\Omega_{1} & =\frac{3}{4} \alpha a_{0}^{2}\left(\tau_{2}\right),
\end{aligned}
$$

following which (33) and (34) take the form

$$
\begin{aligned}
& a_{0,(+1)}=a_{0,(+1)}\left(\tau_{2}\right)=\frac{1}{2} a_{0}\left(\tau_{2}\right) e^{i \phi_{0}\left(\tau_{2}\right)}, \\
& a_{1,(+3)}=a_{1,(+3)}\left(\tau_{2}\right)=\alpha \frac{1}{64} \omega_{0}^{-2} a_{0}^{3}\left(\tau_{2}\right) e^{i 3 \phi_{0}\left(\tau_{2}\right)},
\end{aligned}
$$

where $a_{0,(-m)}\left(\tau_{2}\right)=a_{0,(+m)}^{*}\left(\tau_{2}\right) \quad(m=1,3)$. 
From the results of the foregoing analysis the second-order perturbation equation (23) becomes

$$
\sum_{m=-M}^{+M}\left(1-m^{2}\right) a_{2 .(m)}\left(\tau_{1}, \tau_{2}\right) e^{i m \tau_{0}}=P e^{i \tau_{0}}+Q e^{i 3 \tau_{0}}+R e^{i 3 \tau_{0}}+\text { c.c. }
$$

where

$$
\begin{aligned}
P= & -6 \alpha\left[a_{0,(-1)}^{2} a_{1,(+3)}+2 a_{0,(-1)} a_{0,(+1)} a_{1,(+1)}+a_{0,(+1)}^{2} a_{1,(-3)}\right] \\
& +2 \Omega_{1} a_{1,(+1)}+\Omega_{2} a_{0,(+1)}-i 2 \omega_{0}^{2} a_{0,(+1) \tau_{2} .} .
\end{aligned}
$$

The remaining coefficients $Q$ and $R$ are not important for the order of approximation considered.

For $(m=1)$ Eq. (42) yields

$$
P=0 \text {. }
$$

Substituting (40), (41), (44), and $a_{1 .(+1)}=0$ into (43), and separating the real and imaginary parts result in

$$
\begin{aligned}
\frac{\partial}{\partial \tau_{2}} a_{0} & =0 \\
\frac{\partial}{\partial \tau_{0}} \phi_{0} & =\omega_{0}^{-3}\left[\frac{3}{128} \omega_{0}^{-2} \alpha^{2} a_{0}^{4}-\frac{1}{2} \Omega_{2}\right] .
\end{aligned}
$$

The steady-state solutions of (45) and (46) lead to

$$
a_{0}=\text { constant }, \quad \Omega_{2}=\frac{3}{64} \omega_{0}^{-2} \alpha^{2} a_{0}^{4} .
$$

Substituting the derivatives (39) and (47) into (15) and noting that $\Omega(\varepsilon)=\omega^{2}(\varepsilon)$ lead to

$$
\omega(\varepsilon)=\sqrt{\omega_{0}^{2}+\varepsilon \frac{3}{4} \alpha a_{0}^{2}+\varepsilon^{2} \frac{3}{128} \omega_{0}^{-2} \alpha^{2} a_{0}^{4}+\cdots},
$$

which is the same as the frequency amplitude relationship obtained by Veronis (see Eq. (8)) [5].

For comparison reasons, one can expand (48) into a binomial series and show that it is in full agreement with the relationship given by Nayfeh [6] (see Eq. (7)).

\section{REFERENCES}

[1] A. H. Nayfeh and D. T. Mook, Nonlinear Oscillations, Wiley, New York, 1979

[2] A. S. Atadan and K. Huseyin, A note on "A Uniformly Valid Asymptotic Solution for $\left(d^{2} y / d t^{2}\right)+$ $y=a+\varepsilon y^{2} "$, J. Sound Vibr. 85, 129-131 (1982)

[3] A. S. Atadan and K. Huseyin, An intrinsic method of harmonic analysis for non-linear oscillations (A perturbation technique), J. Sound Vibr. 95, 525-530 (1984)

[4] K. Huseyin and R. Lin, An intrinsic multiple-scale harmonic balance method for non-linear vibration and bifurcation problems, Internat. J. Non-Linear Mech. 26, 727-740 (1991)

[5] G. Veronis, A note on the method of multiple scales, Quart. Appl. Math. 38, 363-368 (1980)

[6] A. H. Nayfeh, Perturbation Methods, Wiley, New York, 1973

[7] K. Huseyin, Elastic Stability of Structures Under Combined Loading, Thesis, University College, London University, England, 1976

[8] K. Huseyin, Nonlinear Theory of Elastic Stability, Noordhoff, Leiden, 1975

[9] K. Huseyin, Multiple Parameter Stability Theory and its Applications. Bifurcations, Catastrophes, Instabilities, Oxford Engineering Science Series, vol. 18, Oxford Science Publications, The Clarendon Press, Oxford University Press, New York, 1986 